\title{
Design of a Laboratory Scale Test Facility for Cross Flow Micro- and Ultra- Filtration Membranes
}

\author{
Muhammad Humble Khalid Tareen ${ }^{1 *}$, Dr. Torsten Brinkmann ${ }^{1}$, Prof. Dr. Samia Khakwani ${ }^{2}$, Kristian Buhr ${ }^{1}$, \\ Holger Pingel $^{1}$, Dr. Juliana Isabel Clodt ${ }^{1}$ and Dr. Nasir M. Ahmad ${ }^{3}$ \\ 1. Helmholtz-Zentrum Geesthacht, Zentrum für Material- und Küstenforschung GmbH, Institute of Polymer Research, \\ Max-Planck-Straße 1, 21502 Geesthacht, Germany \\ 2. Department of Chemistry, The Women University Multan, Pakistan \\ 3. Polymer and Surface Engineering Lab, School of Chemical and Materials Engineering National University of Sciences and \\ Technology, H-12 Sector, Islamabad, Pakistan
}

\begin{abstract}
Systematic UMFF (Ultra- and micro-filtration facility) was developed to investigate the influence of the pressure, flux rate, flux recovery and different solution on different types of membrane. During the study, a control strategy (controlled flow in backflush and filtration process) was formulated, including instrumentation diagrams. After commissioning, calibration was the essential part to run experiments efficiently. The membrane behavior under different conditions was observed by UMFF. Different components were customized and then assembled. In the end different standard membranes were tested to check the performance of the system and to validate the results with independent measurements. During the filtration process, the commercial membranes were test with the ultra-pure (clean) water and different protein solution in order to check the efficiency of UMFF. Fouling behavior of membrane was elaborated and understood by the phenomena of mass transfer. Later, $1 \mathrm{~g} / \mathrm{L}$ concentration of BSA (Bovine serum albumin) was taken for filtration and observed the fouling, which was due to an adsorption of rejected particles on the membrane surface and later, influence the transmembrane pressure. Fluxes of different membranes were analyzed at different flow rates and feed pressures. The membrane behavior towards the back flush method is of great importance, especially flux recovery in order to estimate the efficiency of membrane.
\end{abstract}

Key words: Design of filtration facility, backflush system, permeate flux and flow rate, membrane fouling, flux recovery, protein solution.

\section{Introduction}

Throughout the recent decades, the membrane processes ultra and micro filtrations have played an increasingly important role as a separation technology for various applications. These applications are as diverse as the treatment of surface and waste water, processes in the dairy industry as well as the production of pharmaceuticals [1, 2]. Currently, a new generation of polymeric micro and ultra-filtration membranes is under development at Helmholtz-Zentrum Geesthacht [3]. These membranes

*Corresponding author: Muhammad Humble Khalid Tareen, M.Sc. student, research field: materials science and engineering. are produced from block-copolymers synthesized by controlled anionic polymerization. They exhibit a narrow pore size distribution and high porosity and hence allow for sharp molecular weight cut-offs at high transmembrane fluxes. In order to transfer the potential of these membrane materials from the laboratory into the technical scale, detailed investigations into patterns of the membrane parallel flow, the deposition of cake layers onto the membrane surface and possible cleaning strategies are required $[4,5]$.

For this purpose a new versatile laboratory scale facility is to be developed. The unit is envisaged to operate in a close loop where the feed is supplied from a vessel, led to a feed pump and subsequently enters the membrane module. The retentate is to be throttled 
via a pressure control valve and led back into the feed vessel. In order to cover a wide range of cross flow velocities, an additional pump is supposed to recycle part of the retentate back to the feed. The permeate is also led back to the feed vessel [6-8].

\section{Experimental Section}

\subsection{Materials and Method}

During the experiments, cross-flow filtration was carried out as shown in Fig. 1 in which the scheme of feed, retentate, permeate, backflush flow are mentioned. The cross-flow velocity of the solution over the membrane surface is important parameter to control membrane efficiency.

\subsection{Calibration}

The flow rates change linearly with frequency for a given pressure. If the pressure is increased, the graph will start to deviate from the linear path. The fluctuation of pressure increases at higher values in the device. Fluctuations are noticed at $50 \mathrm{~Hz}$ (3 bars) and $40 \mathrm{~Hz}$ (3 bars) etc. Flow rate and the frequency increase linearly at low pressures like 1.5 bars, but close to 4 bars deviation becomes prominent as shown in Chart 1.

\subsection{Membranes}

PES (Polyethersulfone) and PAN (Polyacrylonitile) are the two commercial membranes, used the in experiments. PES membranes with pore sizes in the range of $100 \mathrm{~nm}$ are used for experiments. They perform well at high flux throughout the entire $\mathrm{pH}$ range. The low level of extractable makes them suitable for environmental analysis [9]. PES membranes have low levels of non-specific protein binding adsorption. PAN membranes are fabricated by

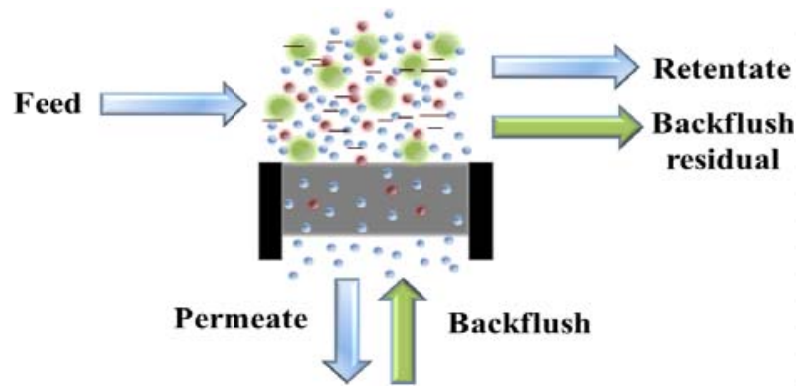

Fig. 1 Flow streams across the membrane in unit cell.

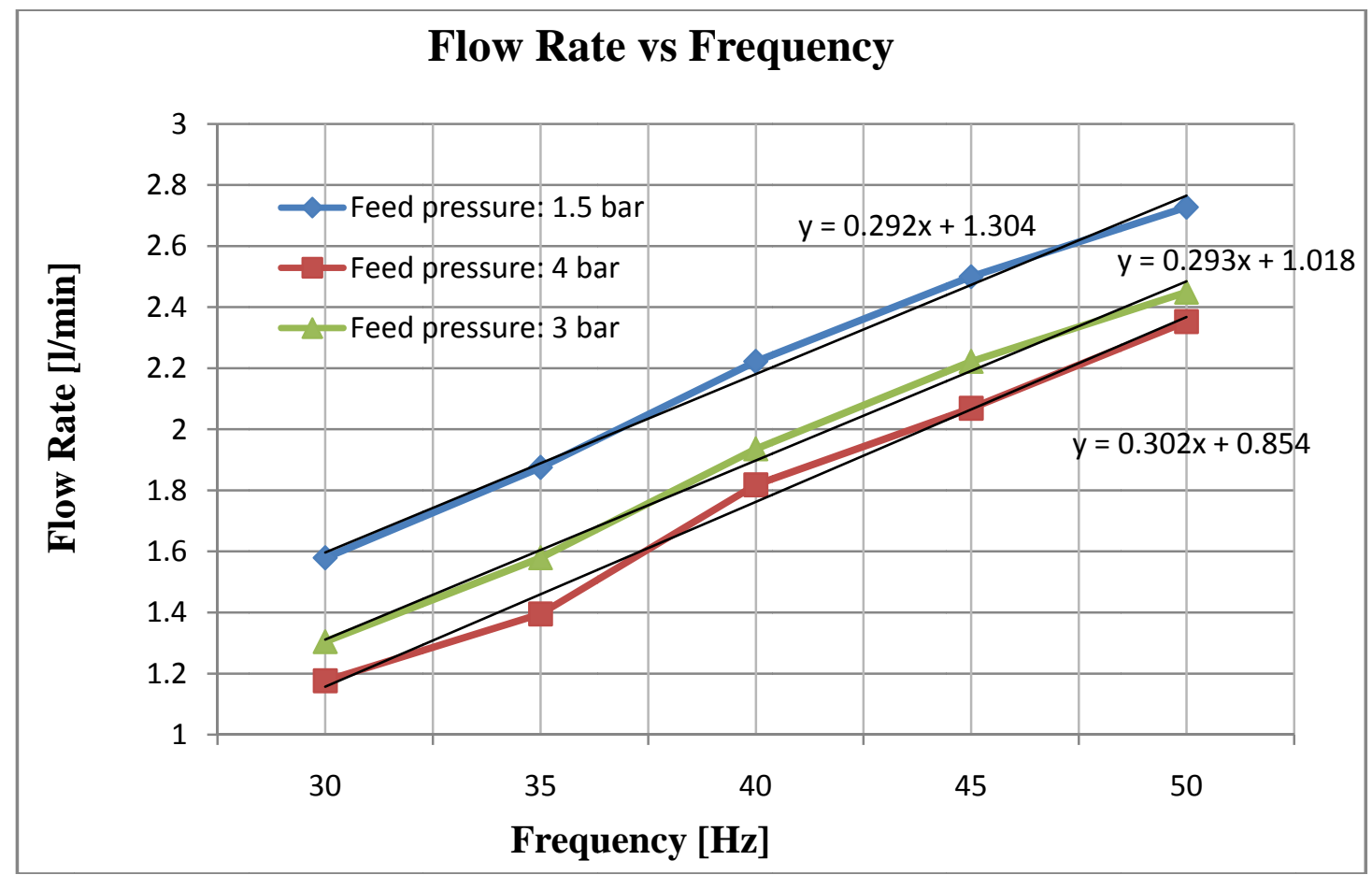

Chart 1 Relation between the frequency and flow rate at different pressures. 


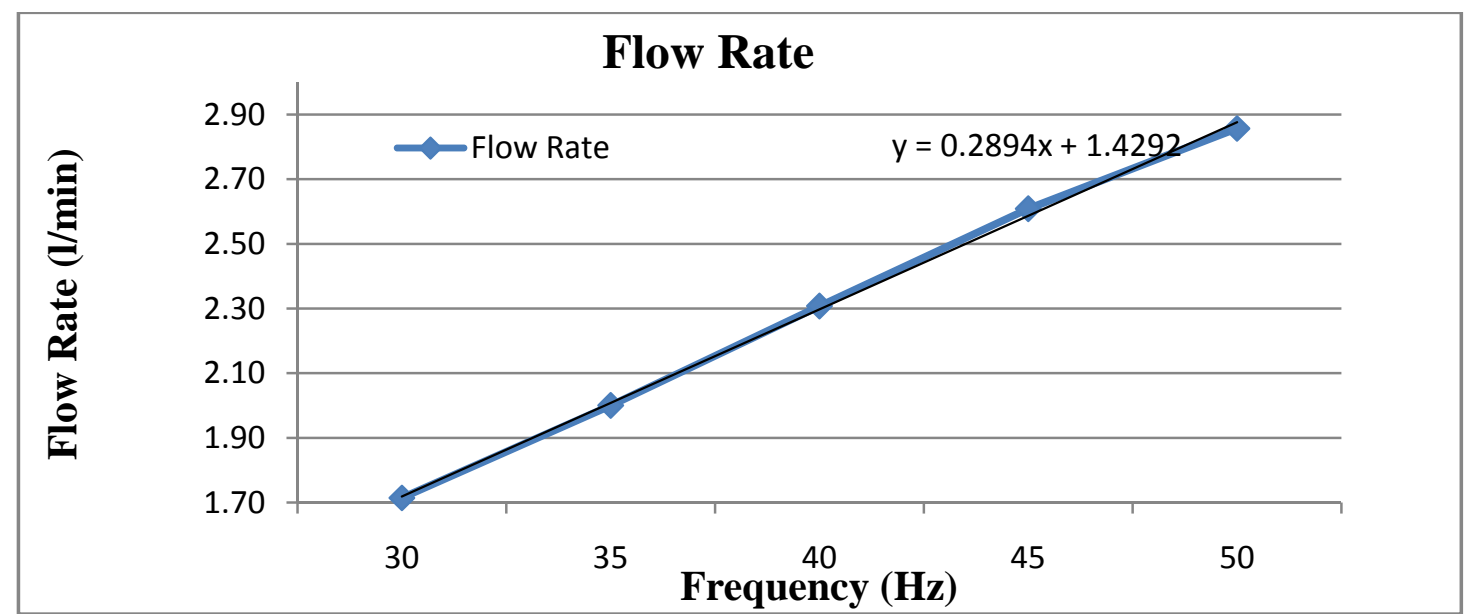

Chart 2 Relation between flow rate and pump frequency.

a phase inversion process. Therefore, different membrane morphologies can be obtained. They vary from large pores to homogeneous micro-porous structures. The permeance of the membranes can be adjusted between 100 up to nearly $2,000 \mathrm{~L} / \mathrm{m}^{2} \cdot \mathrm{h} \cdot \mathrm{bar}$ with a high rejection rate [10].

\subsection{Experimental Procedure}

The feed solution was filled into the feed vessel $\mathrm{V}_{1}$ with a capacity of $5 \mathrm{~L}$. The gear pump $\mathrm{S}_{1}$ was used to supply the feed to the membrane test cell $\mathrm{M}_{1}$ at flow rate of maximum $2.86 \mathrm{~L} / \mathrm{min}$ and a pressure of maximum 4 bar as shown in Scheme 1. Aclose loop system is created to have a same concentration of a solvent. The constant concentration would help to characterize the membrane behavior. Under these conditions different solvents and membranes can be tested. A closed system gives us the behavior of membrane in real condition.

The detailed description of the experimental procedure of a laboratory scale test facility for cross flow of micro and ultrafiltration membrane listed below and details description of system tools are given in Scheme 1. Fig. 2 shows a complete cross flow filtration device.

A circular shape membrane of an area $17.8 \mathrm{~cm}^{2}$ is placed into the test cell along with the supporting material on the lower side and spacer on the top. It is closed after adjusting it with the help of rubber
O-ring.

The valve $\mathrm{C} 1$ has to be checked. It leads the solvent form feed vessel V1 to the pump S1. The C2 and C13 are checked and closed so the liquid cannot leave the system.

The heating or cooling devices are switched on and set to the required temperature of the feed vessel V1. For temperature monitoring place the thermometer.

The feed pump S1 is switched on and the frequency is set with the help of the frequency changer. The required pressure at $\mathrm{p} 1$ is adjusted with the help of pressure regulator (R10) and pressure loss could be observed in the membrane test cell at C15.

The permeate flow rate is measured right after switching on the process to calculate the flux without fouling. 2 to 3 readings should be taken consecutively to observe the variation which would be prominent in the beginning because flux decreases at higher rates. Later on readings after 5 to 7 minutes should be taken. For each reading, the pressure at C14 and C15 should be noted.

The experiment should be run for exactly 1 , 2 or 3 hours. At the end of the experiment, permeate sample form C12 and a retentate sample form C6 are taken. The system is emptied by opening C2 and C13 valves. Pressurized air can be used to empty the system.

The first backflush should take place for 5-15 minutes after first interval of the experiment. 


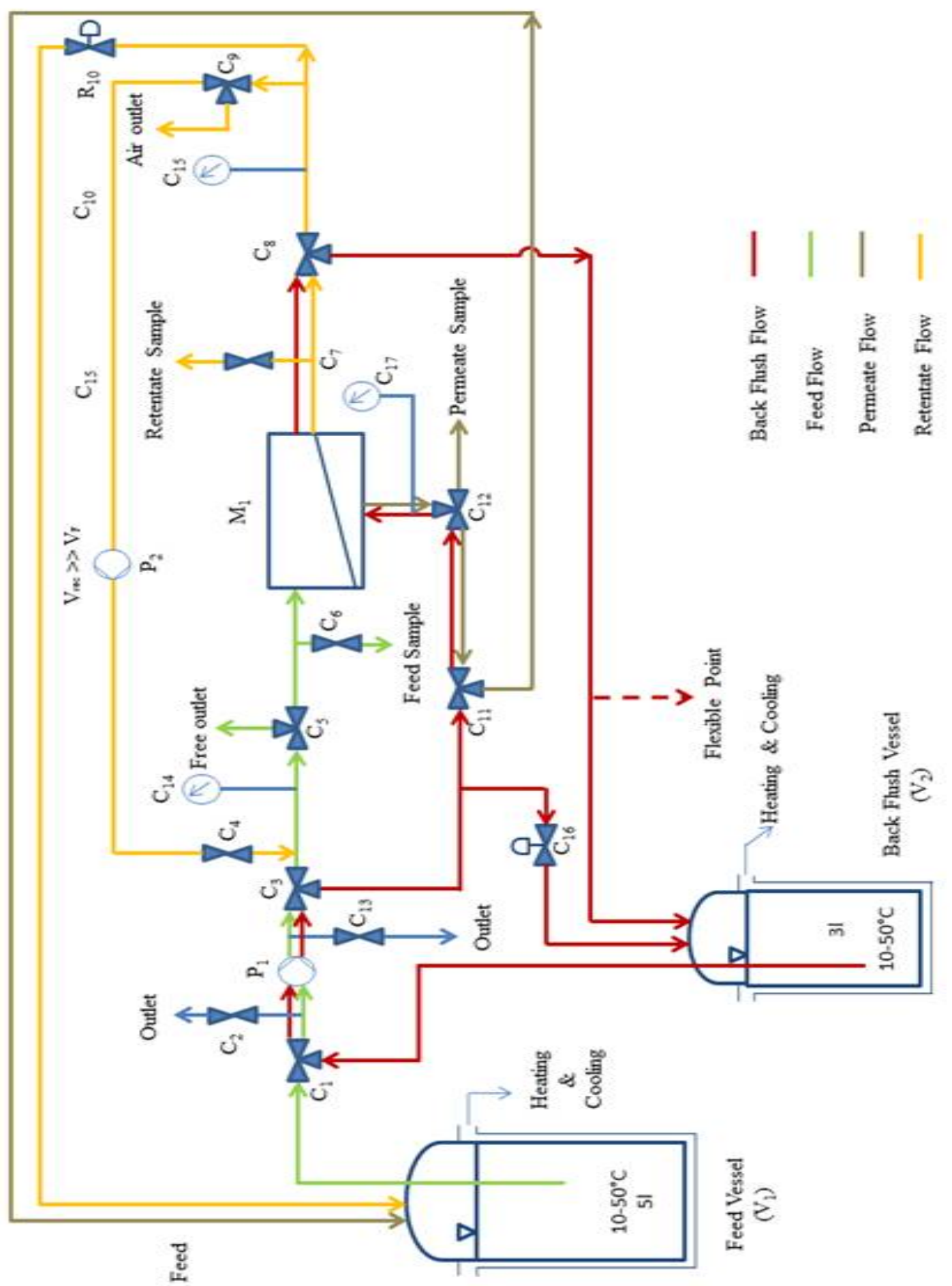

Scheme 1 Complete filtration design's flow diagram.

According to the Scheme 1, C1, C3, C11 and C8 valves should be directed in the way of backflush. C4 and C9 should be closed so flow should not be a part of system while the backflush process is running.

During the backflush process, there is a cross flow.
Permeate should not be the part of the system, otherwise membrane would be blocked from permeate side because of the impure water in a closed loop.

At the end of the experiment, heating and cooling devices are stopped. 


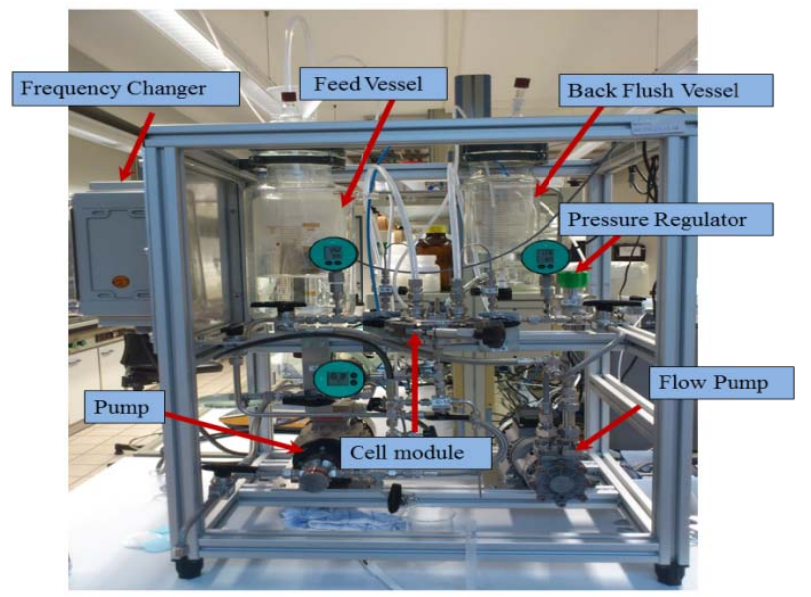

Fig. 2 Micro- and ultra- filtration facility.

Both feed pumps S1 and flow pump S2 are stopped and the solvent first from feed vessel $\mathrm{V} 1$ and the backflush vessel V2 is removed.

The membrane test cell is opened and membrane is removed carefully without damaging. Unwanted protein and other solute particles are removed from the system by running with ultra-pure water [8].

\section{Results and Discussion}

\subsection{Experiment 1}

The First experiment was performed with the polyacrylonitrile membrane, where the solvent was ultra-pure water in the feed vessel $V_{1}$. The duration of the experiment time was 5 hours. The feed pressure was between 3.12 bars to 3.16 bars and the feed flow rate was up to $2.4 \mathrm{~L} / \mathrm{min}$. The trans-membrane pressure reached up to a maximum of 2.11 bars. At high pressures fluctuation increases in the system. Feed pump frequency was $50 \mathrm{~Hz}$. A small deviation in the flux was present, which confirmed the literature [12] as well. The reasons are attributed to other particulate matter accumulated from the surrounding like pipes etc.

In Chart 3, it was shown that the flux values almost remain constant, during filtration, at the high pressure range. There was only a small $9.17 \%$ decline in flux. This small amount of deviation was due to impurities from pumps or the dust particles from surrounding. At higher pressure there was a larger decline in the presence of the other solvents. Experimental values were also compared with Literature in 2.3 .

\subsection{Experiment 2}

The second experiment was conducted on the polyethersulfone membrane with the ultra-pure water as feed solvent 3 backflushes were performed after one hour of operation and the membrane behavior was observed. The feed pressure was 1.13 bars. The flow

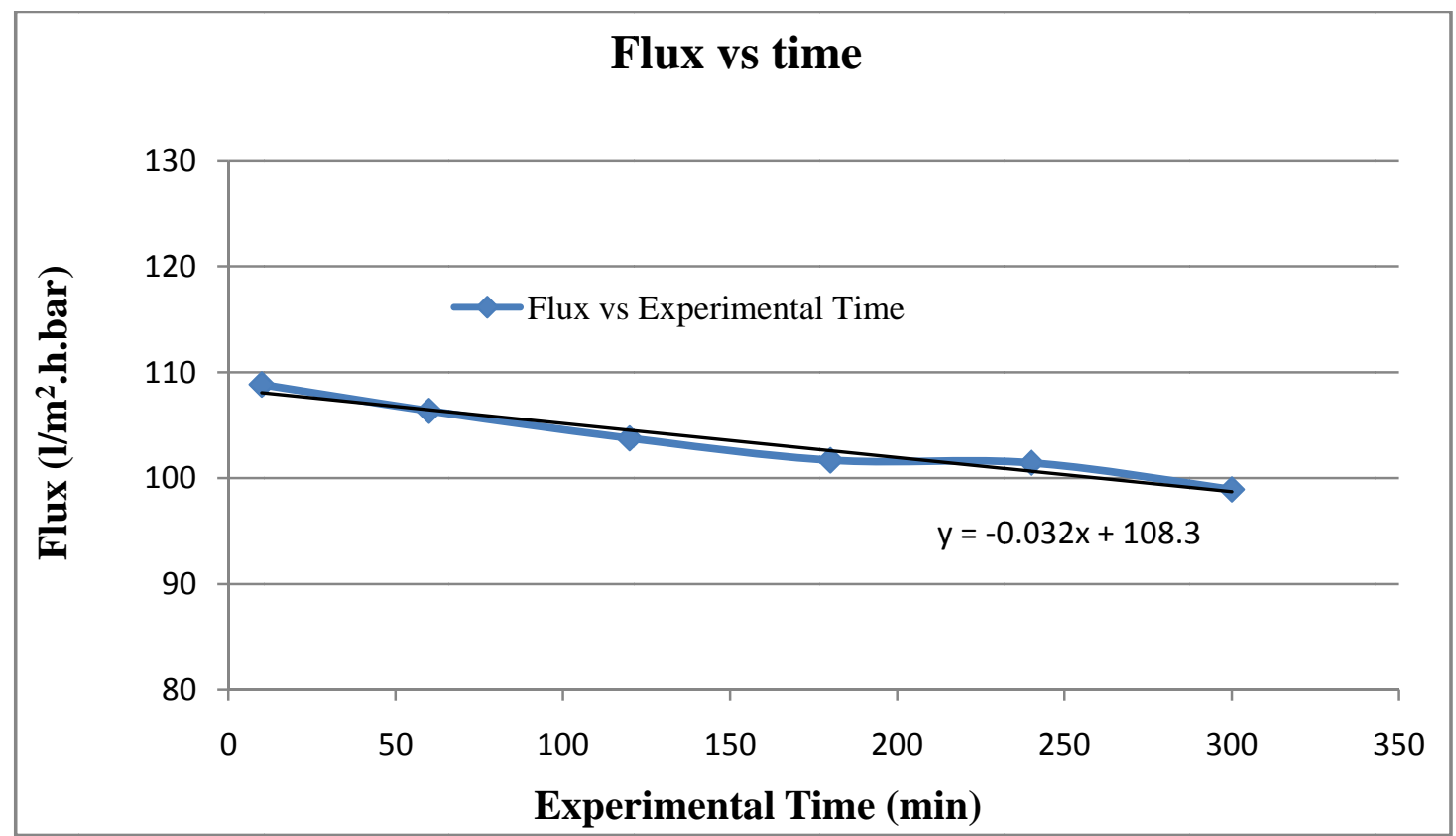

Chart 3 The graph shows the values of flux depending on time for the first experiment. 
rate over the membrane was $2.86 \mathrm{~L} / \mathrm{min}$. During the backflush process, filtration process was stopped and the ultra-pure water will run from permeate to the retentate side, whereas feed side was remain closed. The backflush pressure was 1.36 bars and pump frequency was $30 \mathrm{~Hz}$. Before applying the backflush, the pressure range and the proper support of the membrane had to be verified, otherwise membrane would have ruptured.

The initial decline in flux observed from Chart 4 . During the first backlash, the clean water flux decreases slightly up to $35 \%$ and $15.39 \%$ of flux decline in second and third backflush. The flux decline was due to impurities in the system. After applying backflush for 10-15 minutes with ultra-pure water, the flux reached the initial value. In other words, flux recovery was $100 \%$ as shown in Table 1 . The same behavior was seen after the second backflush. There was no significant flux recovery after the backflush as ultra-pure water was used in the filtration process.

Experimentally, it was analyzed that even with the ultra-pure water, there was a small variation in the flux due to the impurities still present in the system like pipes, vessels etc. Experimental values were also compared with literature values [11].

\subsection{Experiment 3}

In this experiment, polyethersulfone membrane was used with $1 \mathrm{~g} / \mathrm{L}$ solution concentration of BSA (Bovine serum albumin) as feed solvent. Two backflushes were performed after 1 hour interval of filtration. The feed pump frequency was $30 \mathrm{~Hz}$, feed pressure was 1.13 bars and feed flow rate was 1.71 $\mathrm{L} / \mathrm{min}$. The first reading was taken with ultra-pure water in order to have a reference value and can be compared with another solution, like in our case BSA solution.

Initially, flux decreased drastically and kept on decreasing in a progressive manner as shown in Chart 5.

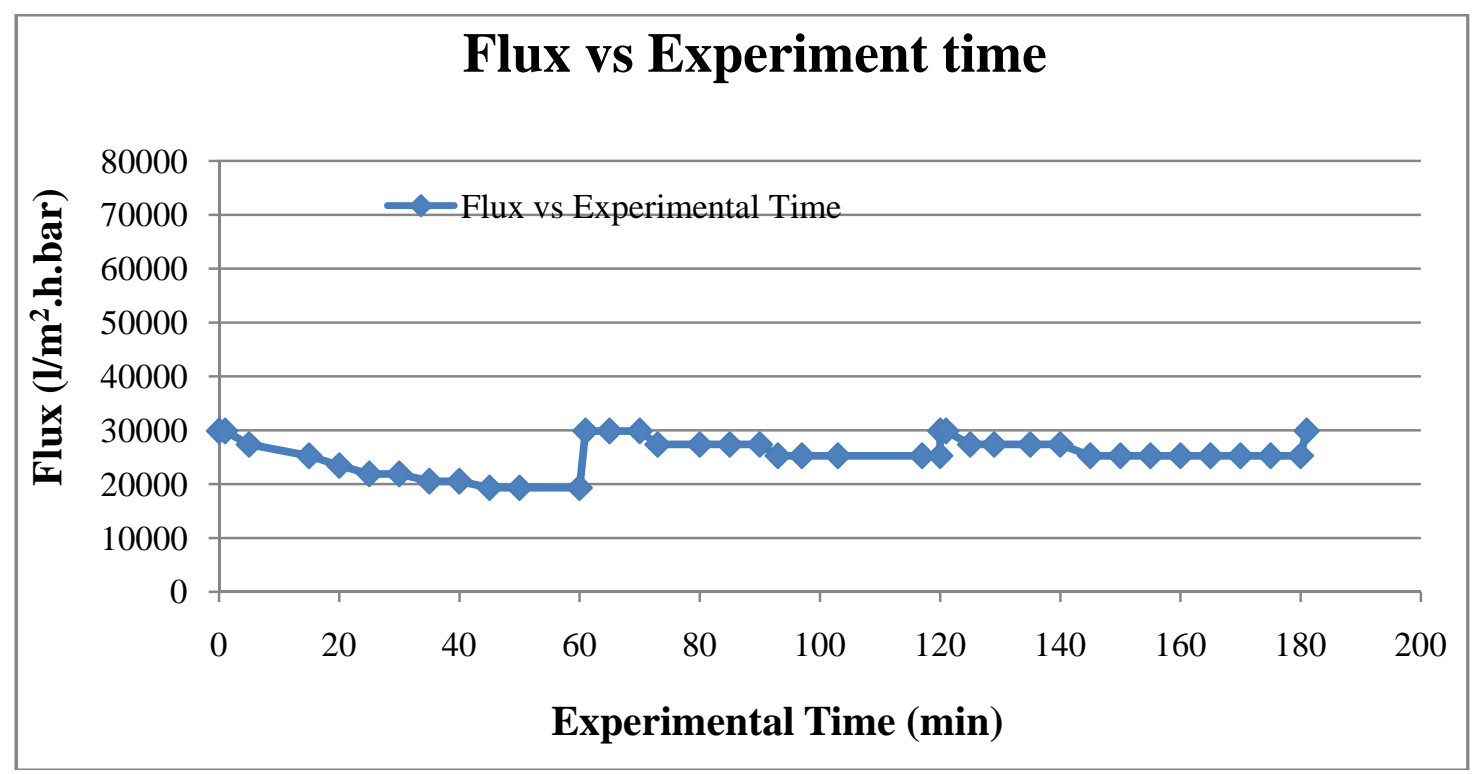

Chart 4 The graph shows the values of ultra-pure water flux depending on time and three backflush cycles.

Table 1 Flux percentage analysis of experiment 2, before and after the backflushes.

\begin{tabular}{lllll}
\hline Flux (\%) & First hour & Second hour & Third hour & Avg. \\
\hline Minimum & 65 & 85 & 85 & 78 \\
Decline & 35 & 15 & 15 & 22 \\
& After backflush & & & 100 \\
Recovery & 100 & 100 & & 100 \\
\hline
\end{tabular}




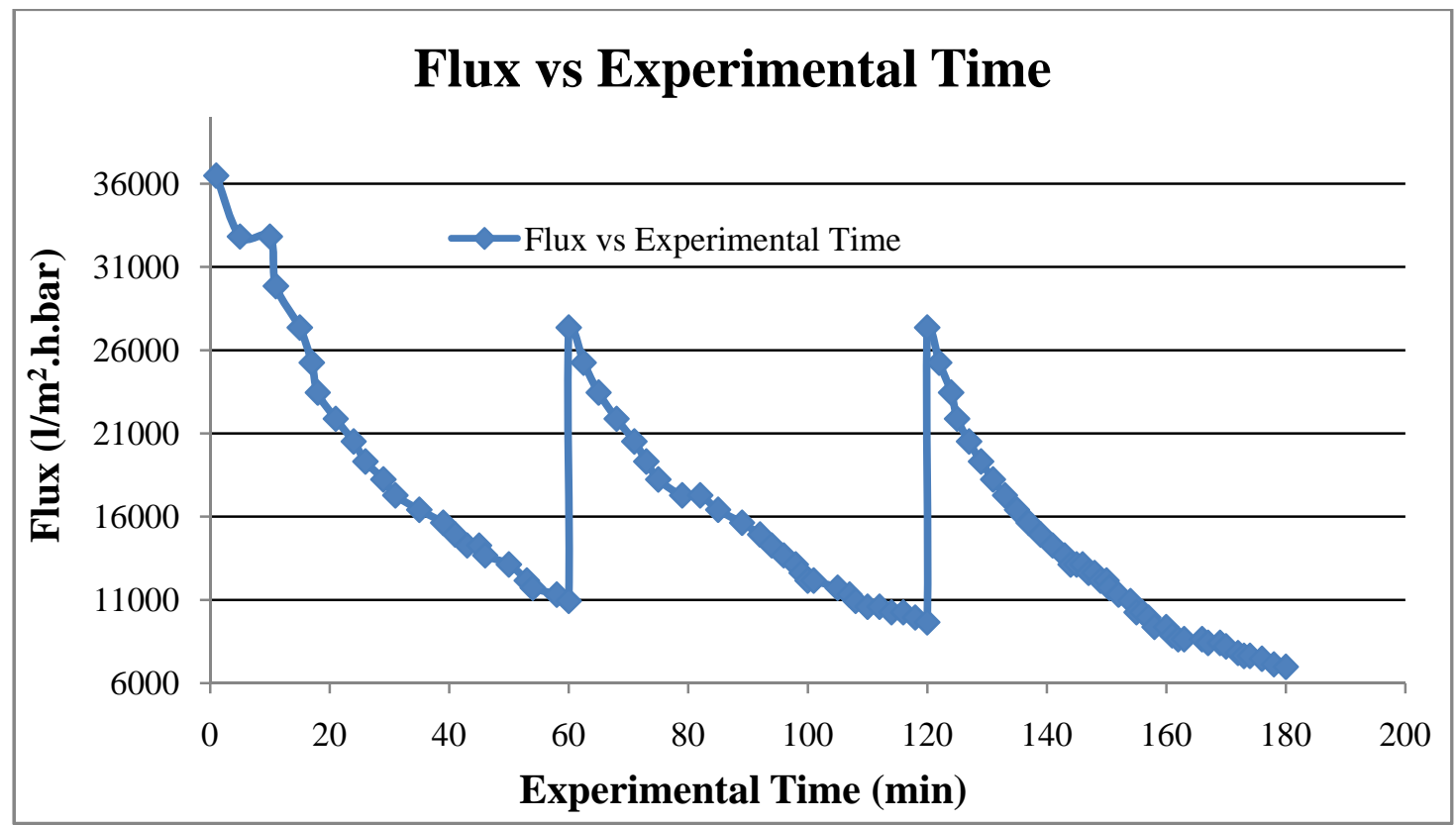

Chart 5 The graph shows the values of BSA flux depending on time and three backflush cycles.

Table 2 Flux percentage analysis of experiment 3, before and after the backflushes.

\begin{tabular}{lllll}
\hline Flux(\%) & First hour & Second hour & Third hour & Avg. \\
\hline Minimum & 34 & 37 & 26 & 22 \\
Decline & 66 & 63 & 74 & 78 \\
& After backflush & & & 84 \\
Recovery & 84 & 84 & & 84 \\
\hline
\end{tabular}

Using BSA solution, the flux decline was much more as compared to the ultra-pure water experiment. Two backflushes were applied with the ultra-pure water as conducted in the experiment 2 and same precautions were taken. In the first hour of operation, the flux declined up to 66 percent as shown in Table 2. After applying the first backflush, the flux recovery was $83 \%$, but not fully recovered because of the permanent fouling (chemical fouling) which was $17 \%$ as shown in Table 2. During the second hour of operation, flux decline was almost same as in the first hour. The same behavior was seen after the second backflush i.e. flux recovery was $83 \%$. The flux decline during the third hour of operation was $11 \%$ more as compared to second hour. After the second backflush, the flux increased more as compared to the first backflush because of the less chemical fouling. After the second hour, flux decline $10.84 \%$ more as compared to the first hour.
In this experiment, flux decline was $30.71 \%$ higher as compared to the experiment 2 with ultra-pure water. In last 10 minutes, the reduction in the flux again increased to some extent as shown in last section of chart

\section{Conclusions}

A laboratory scale test facility for cross flow filtration was successfully constructed and operated in the current study. The UMFF (Ultra- and micro-filtration facility) was capability for the separation of desired particles such as protein, like BSA protein , bacteria or PEG-molecules.

First, the concise instrumentation diagram was devised to construct a test facility which meets the standard, like minimum fouling, permeate flux, cross-flow operation mode etc, required for the experiments. A detailed control strategy was developed to reduce the pressure loss and to perform 
other functions like backflush.

Finally, the membrane behavior was analyzed by the unit for flow rates, pressures, flux and fouling.

\section{Acknowledgment}

First, I am pleased to have financial and technical support provided by the Helmholtz Zentrum Geesthacht for giving me the opportunity to pursue work on this project in the department of Process Engineering, Institute of Polymer Research. Secondly, I would like to thank the chair of Chemical physics and Materials Sciences, University of Augsburg for allowing me to work under their supervision. I attribute the level of my study to their encouragement and effort and without them this research, too, would not have been completed or written.

Last but not the least, I would like to thank my family, my parents Prof. Qudsia Khakwani and Khalid Tareen, for supporting me spiritually and believing in me throughout my life.

\section{References}

[1] Elford, W. 1937. "Principles Governing the Preparation of Membranes Having Graded Porosities.” In The Properties of "Gradocol" Membranes as Ultrafilters, Transactions of the Faraday Society. 33: 1094-104.

[2] Sidney, L. and Srinivasa, S. 1963. "Sea Water Demineralization by Means of an Osmotic Membrane.” American Chemical Society. 38 (II): 117-32.

[3] Baker, R. W. 2000. “Membrane Technology.” Wiley Online Library.

[4] Zsigmondy, R. and Bachmann, W. 1918. "Ueber neue Filter.” Z. Anorg. Alg. Chem. 103: 119-28.

[5] Ferry, J. D. 1936. "Ultrafilter Membranes and Ultrafiltration.” Chemical Reviews 18: 373-455.

[6] Van Reis, R. and Zydney, A. 2007. "Bioprocess Membrane Technology.” Journal of Membrane Science 297 (1): 16-50.

[7] Scharnagl, N. and Buschatz, H. 2001. "Polyacrylonitrile (PAN) Membranes for Ultra- and Microfiltration.” Desalination 139 (1): 191-8.

[8] Walker, R. 1982. Recent Developments in Ultrafiltration of Electrocoat Paint, Electrocoat.

[9] Description of Polyethersulfone (PES) Membrane Filter. Sartorius.

https://www.sartorius.com/en/product/product-detail/1 5406-47-n/.

[10] B.G.a.-F.a.M.a.S. José Miguel Arnal, 2011. Membrane Cleaning, Expanding Issues in Desalination, Intec.

[11] PES Specifications. PALL Corporation. 